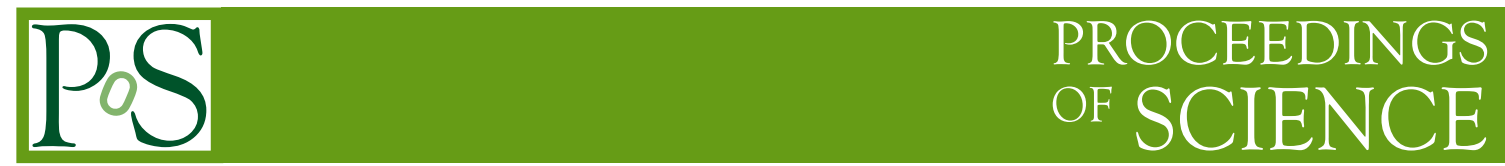

\title{
Higgs fields, Yang-Mills Quantized theories and the Cohomological origine of the mass
}

\section{Joseph Kouneiher*}

Université de Nice Sophia Antipolis

E-mail: joseph.kouneiher@unice.fr

In this talk we want to show how the mass can enter Yang-Mills theories through central (pseudo) extensions of the corresponding gauge group. This mechanism does not involve extra (Higgs) scalar particles and could provide new clues for the better understanding of the nature of the Symmetry Breaking Mechanism. In this talk we are going to outline the essential points of this approach.

Frontiers of Fundamental Physics 14 - FFP14,

15-18 July 2014

Aix Marseille University (AMU) Saint-Charles Campus, Marseille

* Speaker. 